\title{
TV Guided Missile Target Image Processing Improvement
}

\author{
He Jingfeng*, Ji Ming, Xiao Ruliang \\ College of Control Engineering, Xijing University, Xian, 710123, China \\ email: 573825625@qq.com
}

Key Word: Image segmentation; TV guided missile; Ostu; Genetic algorithm

\begin{abstract}
In using the TV-guided missiles to attack targets process, the target image segmentation process directly affects the attack effect. So, choose a suitable image segmentation methods to ensure high quality target image processing speed, real-time, is particularly important. This paper introduces a method which combines the advantages of the genetic algorithm with the advantages of the fast optimization principle and the method of Ostu, to improve the method of Ostu, and Simulation on the simulation platform. The simulation results show that the improved algorithm is efficient and effective. Simulation results show that the improved Ostu good treatment effect and the calculation speed of the algorithm is effective.
\end{abstract}

\section{Introduction}

TV guidance is the technology to control and guide the missile to the target by TV. The television guidance system is composed of a television camera, an autopilot, a television transmission system, a receiving system, an image information processing system, a tracking servo system, etc .TV guidance has two ways, one is TV command guidance, this way is installed in guiding head camera to capture target image processing, send to the operator, by the operating personnel targets are selected according to the captured image signal, wireless command control seeker attack on the target. The other is a television homing guidance. This is the camera to capture the image signal processing, sent directly to the guidance device, guided missile attack. TV guided missile is flexible, rapid response, not subject to geographical features, in the air to ground attack are widely used. Target acquisition and tracking ability of TV guided missile seeker is mainly determined by the missile borne computer to the target image segmentation quality and the recognition speed. However, the missile computer on the calculation speed of image is mainly determined by two factors, one is the calculation speed of the computer system itself, the two is the target image segmentation algorithm. Therefore, in order to take advantage of segmentation algorithm in air combat is necessary for the missile borne computer of target image research.

\section{Research Status Of Image Segmentation}

At present, there are many methods of image segmentation, Commonly used segmentation methods have three types of edge detection, region tracking and threshold ${ }^{[1]}$. Threshold is simple, effective, simple, fast and so on. It is the first choice in image processing. In order to study the image segmentation algorithm of TV guided missile, this paper applies the advantages of parallel search and global optimization to Ostu method of the quality of the threshold image segmentation. Combining the advantages of the two methods to improve the Ostu method, improve the speed of the search for the optimal threshold value, and effectively improve the efficiency of image segmentation.

\section{Improvement Of Otsu}

The Principle Of Threshold Image Segmentation. The gray level range of image $f(x, y)$ captured by video guided missile seeker is as a threshold value $G=\{0,1, \bullet \bullet, L-1\}, t \in G, \mathrm{~B}=\left\{\mathrm{b}_{0}, \mathrm{~b}_{1}\right\}$ is a pair of two values, and $b_{0}, b_{1} \in G$. Threshold t settings can be obtained by the image of a two value image 
$\mathrm{ft}$, that is

$$
f_{t}(x, y)= \begin{cases}b_{0} & f(x, y)<t \\ b_{1} & f(x, y) \geq t\end{cases}
$$

It can be seen from the above formula, how to select the optimal threshold value $t$ in the range of $\mathrm{T}$ is the key to the threshold image segmentation method. Threshold image segmentation method has global threshold and local threshold method. The difference between global threshold and local threshold method is in the image processing, global threshold method is the whole image as a whole to select a threshold value $\mathrm{T}$, and bureau of limit method is according to the gradation of the image is divided into several sub plots were selected for different threshold value t. Ostu is one of the most widely used image processing methods in the global threshold method.

\section{Otsu Basic Principle ${ }^{[2]}$}

Assuming that the image captured by the guidance of TV guided missile seeker has $\mathrm{N}$ pixels , pixel gray level $L$, the image gray level for $i$ all the pixel number of $n_{i}$, in the image gray level for the number of pixels i accounted for the probability of the whole image:

$$
P_{\mathrm{i}}=n_{\mathrm{i}} / N \quad i=1,2, \ldots, L-1
$$

The probability of all gray level appearance is 1 ,that is:

$$
\sum_{i=0}^{L-1} p_{i}=1
$$

The meaning of image segmentation is to separate the target from the original image containing the background. Therefore in the image processing, the image is divided into two parts: $\mathrm{C}_{0}$ is the target region, $\mathrm{C}_{1}$ is background region. $C_{\mathrm{o}}=\{0,1, \mathbf{9 g g} t\}, c_{1}=\{t+1, t+2, \cdots L-1\}$. The image threshold value is $t$, then the gray mean value of the image is:

$$
u_{t}=\sum_{i=0}^{L-1} i p_{i}
$$

The mean of the target and the background are:

$$
\begin{aligned}
& u_{0}=\sum_{i=0}^{t} i \operatorname{Pr}\left(c_{0} \mid i\right)=\sum_{i=0}^{t} i p_{i} / \omega_{0} \\
& m_{1}=\sum_{i=t+1}^{L-1} i \operatorname{Pr}\left(c_{1} \mid i\right)=\sum_{i=t+1}^{L-1} i p_{i} / \omega_{1}
\end{aligned}
$$

Among them:

$$
\begin{aligned}
& \omega_{0}=\operatorname{Pr}\left(c_{0}\right)=\sum_{i=0}^{t} p_{i} \\
& \omega_{1}=\operatorname{Pr}\left(c_{1}\right)=\sum_{i=t+1}^{L-1} p_{i}=1-\omega_{0}
\end{aligned}
$$

All available:

$u_{t}=\omega_{0} u_{0}+\omega_{1} u_{1}$

Therefore, the inter class variance is: 


$$
\begin{aligned}
& \sigma_{b}^{2}(t)=\omega_{0}\left(u_{0}-u_{t}\right)^{2}+\omega_{1}\left(u_{1}-u_{t}\right)^{2} \\
& =\omega_{0}\left(u_{0}^{2}+u_{t}^{2}\right)+u_{t}^{2}\left(\omega_{0}+\omega_{1}\right)^{2}-2\left(\omega_{0} u_{0}+\omega_{1} u_{1}\right) u_{t} \\
& =\omega_{0} u_{0}^{2}+\omega_{1} u_{1}^{2}-u_{t}^{2} \\
& =\omega_{0} u_{0}^{2}+\omega_{1} u_{1}^{2}-\left(\omega_{0} u_{0}+\omega_{1} u_{1}\right)^{2} \\
& =\omega_{0} u_{0}^{2}\left(1-\omega_{0}\right)+\omega_{1} u_{1}^{2}\left(1-\omega_{1}\right)-2 u_{1} u_{0} \omega_{1} \omega_{0} \\
& =\omega_{1} \omega_{0}\left(u_{0}-u_{1}\right)^{2}
\end{aligned}
$$

Let $\sigma_{b}^{2}\left(t^{*}\right)$ be between class variance, the optimal threshold $t^{*}$ is to make the maximum value of the corresponding gray level ${ }^{[3,4]}$. That there is:

$$
\sigma_{b}^{2}\left(t^{*}\right)=\max _{t=G} \sigma_{b}^{2}(t)
$$

\section{Improved Steps For Otsu}

As TV seeker for guided missile shot image target accounts for the proportion of very small, gray information of the target is not enough to affect the whole image gray information. If you want to separate the target from the background image in the Central District, when the target images in the whole image occupy a certain proportion, we can use the between class variance method to image processing, use Otsu method for image processing, need an optimal threshold value $t^{*}$ ensure the between class variance function maximum. The quality of image segmentation and the speed of image processing directly depend on whether the threshold value $t^{*}$ is the best choice. In the TV guided missile, both the results of the image processing and the real-time problem must be considered, after all, these two indicators are related to the target tracking attack effect. Therefore, algorithm of the optimal threshold selection of very important, traditional optimization methods and steps of many large amount of computation, considering the genetic algorithm can be within the scope of global search and automatic selection of threshold value, greatly shortened the time. In order to take into account the above indicators, the Otsu algorithm is introduced into the genetic algorithm to find the optimal principle, determine the best search space, find the optimal threshold valuet*.

Genetic algorithm ${ }^{[5]}$ was first proposed by Professor J. Holland in the United States in 1975. It is a global search algorithm for random search through the space of the target. The basic idea of this algorithm is the simulation of natural selection and genetics theory, on the basis of the principle of survival of the fittest. Genetic algorithm is also used in the field of Computer Science in the field of artificial intelligence to solve the optimization of a search heuristic algorithm is an evolutionary algorithm ${ }^{[6]}$.

The solution of Otsu algorithm is to select the optimal threshold value, so that the maximum variance between the target and the background is the biggest, which can achieve the cutting of the background image and the target image, but the method is greatly influenced by the speed of the computer. Considering the genetic algorithm can search in the global, the threshold value to carry out intelligent screening, so we will search principle of genetic algorithm introduced the between class variance method, so as to achieve the improvement of the Otsu algorithm, not only meet the image segmentation effect, and also shorten the image processing time, with TV guided missile borne computer calculation speed to meet the requirements.

In the genetic algorithm based on the improved Otsu image segmentation steps are as follows: step 1: Determine the decision variables and various constraints, that is, to determine the individual expression of the $\mathrm{X}$ and the problem of the solution space ${ }^{[7]}$.Decision variables are the required op- 
timal threshold value $t^{*}$, because the image gray value captured by the TV guided missile is in the range of $0 \sim 255$, so the optimal threshold value $t^{*}$ is the integer value in the range.

step 2: Determining fitness function. The proportion of variance between OSTU background image and target image is proportional to the accuracy of segmentation. The fitness function value is greater, indicating the foreground and the background image difference is bigger, the better segmentation effect ${ }^{[8]}$. We can use the corresponding image between class variance to set the function formula ${ }^{[9]}$. Fitness function:

$$
g(t)=\sigma_{b}^{2}(t)=\omega_{0} \omega_{1}\left(u_{0}-u_{1}\right)^{2}
$$

Step3:Select operation. Select operation using the proportional selection operator.

Step4:Crossover operation. Selection probability is 0.8 single point crossover operator.

Step5:Mutation operation. Uniform mutation operator with a probability of 0.008 .

Step6:Set running parameters and end conditions. In the operation, taking into account the effect of image segmentation and image processing speed, the number of entries and the evolution algebra are set to 10,20 . The algebraic upper bound is set to the end condition, and the upper bound is stopped.

In the genetic algorithm based on the improved Otsu algorithm flow chart as shown in figure 1.

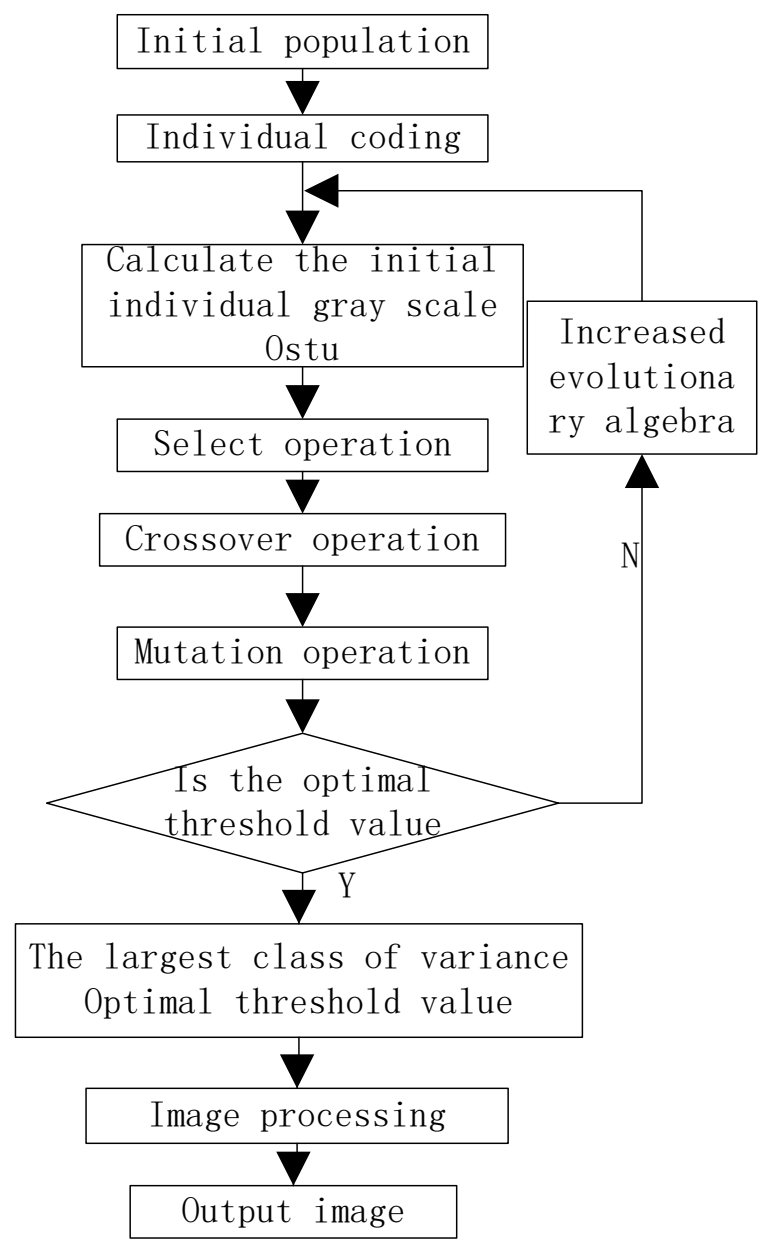

Fig. 1 flow chart of improved Otsu algorithm

\section{Simulation Result Analysis}

In order to verify the improved genetic algorithm to the Otsu method, we use the improved inter class variance method to image processing experiments on the ground simulation training platform 
of TV guided missile. The following is the original image captured and improved Otsu class variance method between the segmentation of the target image.

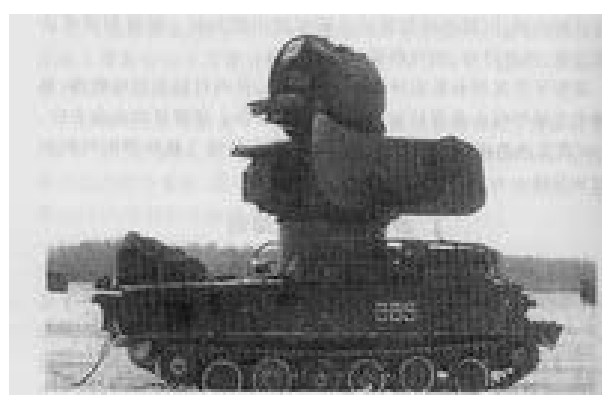

(a)Original

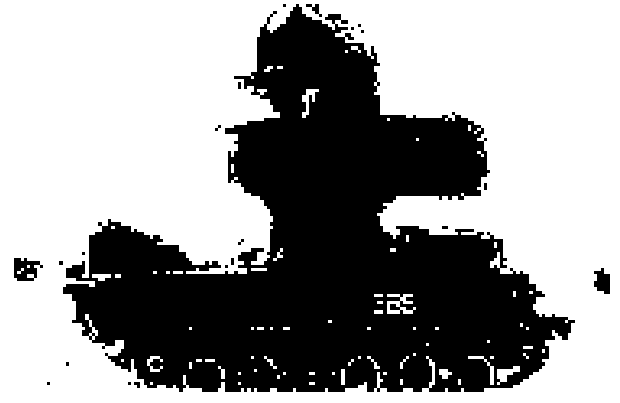

(b)Improved algorithm segmentation graph

Fig. 2 Comparison of segmentation

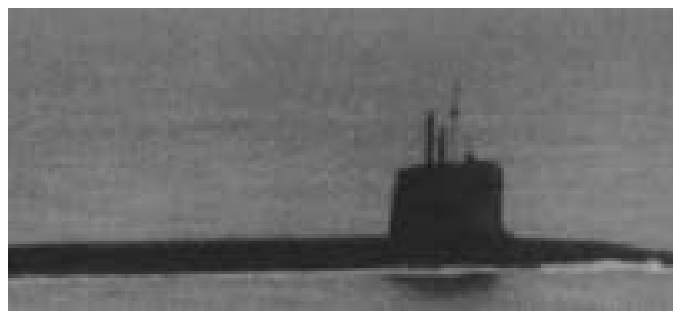

(a)Original

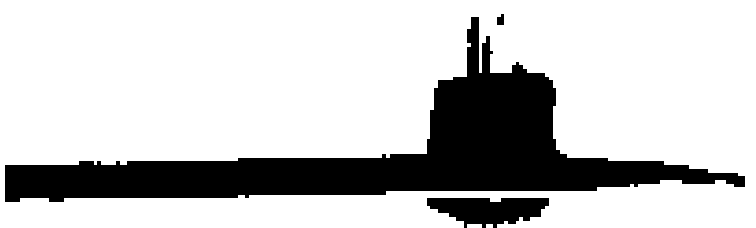

(b)Improved algorithm segmentation graph

Fig. 3 Comparison of segmentation

From Figure 2, and figure 3 image segmentation comparison chart can be seen, after using the improved Otsu method, good segmentation effect, with clear target characteristics, modified between class variance method with the result of the meet TV guided missile target tracking of image decomposition, while also taking into account the bomb load calculation of image processing speed requirements.

\section{Summary}

According to the simulation results, it is showed that the Otsu method combined with genetic algorithm is applied to image processing, fast speed, clear image, good segmentation effect and good real-time performance. To improve the recognition of TV guided missile, the target tracking accuracy requirements. Provides a good method for the study of TV guided missile.

\section{References}

[1] Zhou Ming, Sun Shudong. Principle and application of genetic algorithm M. Beijing: National Defence Industry Press, 2002

[2] Ren Xiaona. Based on the algorithm research and application [J]. Electronic design engineering for automatic selection of image threshold of Otsu algorithm, 2015,23 (11), 75-77

[3] Hou Gexian. Genetic algorithm and its application in tracking system [D] Xi'an: Xi'an Electronic and Science University, 1998

[4] Cui Yi. Digital image processing technology and application [M]. Beijing: Electronic Industry Press, 2001

[5] Peng Xuan. Genetic algorithm and its application in image segmentation [D]: Changchun: Jilin University, 2006

[6]Tan Xiaohong. Multi factor slender rod multi cavity mold injection molding process integrated 
multiobjective optimization [D]. Zhenjiang: Jiangsu University, 2013

[7] Yang Li. Ridgelet redundant dictionary and genetic evolution of compressed sensing reconstruction based on [D]. Xi'an: Xi'an Electronic and Science University, 2012

[8] Wang, Qun Feng Xu Yinghui. Combined with improved genetic algorithm and local threshold method of fingerprint image segmentation [J]. Automation and information engineering, 2015,36 (1). 12-17

[9] Luo Lixia. Ostu image segmentation algorithm based on the method [J]. Journal of Hebei North University (NATURAL SCIENCE EDITION), 2014,30 (6), 29-33

基金项目：西京学院科研基金资助项目(XJ140120);国家大学生创业实践项目 (201512715801) 